\title{
Editorial
}

\section{Silicon Photonics}

\author{
Pavel Cheben, ${ }^{1}$ Richard Soref, ${ }^{2}$ David Lockwood, ${ }^{1}$ and Graham Reed ${ }^{3}$ \\ ${ }^{1}$ Institute for Microstructural Sciences, National Research Council of Canada, Ottawa, ON, Canada K1A OR6 \\ ${ }^{2}$ Air Force Research Laboratory, Sensors Directorate, Electromagenetics Technology Division, \\ Hanscom Air Force Base, Massachusetts 01731, USA \\ ${ }^{3}$ Department of Electronic Engineering, School of Electronics and Physical Sciences, University of Surrey, Guildford, \\ Surrey GU2 7XH, UK
}

Correspondence should be addressed to Pavel Cheben, pavel.cheben@nrc-cnrc.gc.ca

Received 17 June 2008; Accepted 25 June 2008

Copyright (c) 2008 Pavel Cheben et al. This is an open access article distributed under the Creative Commons Attribution License, which permits unrestricted use, distribution, and reproduction in any medium, provided the original work is properly cited.

With the goal of developing photonic components that are factory-compatible with silicon microelectronic integrated circuits and optical integrated circuits, silicon photonics has been the subject of intense research activity in both industry and academia. Silicon is an excellent material for confining and manipulating light at the submicrometer scale, and possesses the added advantage of leveraging the enormous manufacturing infrastructure developed by the silicon microelectronics industry. Silicon optoelectronic integrated devices have the potential to be miniaturized and mass-produced at affordable cost for many applications and markets, including telecommunications, optical interconnects, medical screening, and biological and chemical sensing. Recent developments in diverse areas, such as light sources, modulators, switches, detectors, photonic crystals, waveguide structures, resonators, sensors, and various subsystems, indicate that Si photonics is an extremely active, and now, firmly established research field.

The aim of this special issue is to document some of the remarkable recent progress in silicon photonics from academic and industrial viewpoints and thereby point to future trends in this rapidly evolving field. All the articles in this issue are invited review or invited research papers by leading authorities and research groups in this field from universities, industry and government laboratories.

The invited papers of this special issue are the following.

"The achievements and challenges of silicon photonics" by R. Soref.

"Synthesis of planar reflective gratings for silicon interconnects" by R. Millett et al.

"High-speed near infrared optical receivers based on Ge waveguide photodetectors integrated in a CMOS process" by G. Masini et al.
"Light emission from rare-earth doped silicon nanostructures" by J. Li et al.

"Development of silicon photonics devices using microelectronic tools for the integration on top of a CMOS wafer" by J. M. Fedeli et al.

"Developments in gigascale silicon optical modulators using free carrier dispersion mechanisms" by J. Basak et al.

"Photonic integration on hybrid silicon evanescent device platform" by $\mathrm{H}$. Park et al.

"Subwavelength grating structures in silicon-on-insulator waveguides" by J. H. Schmid et al.

"Hybrid silicon photonics for low-cost high-bandwidth link applications" by B. Jonathan Luff et al.

"On-chip all-optical switching and memory by silicon photonic crystal nanocavities" by M. Notomi et al.

"Optical filters utilizing ion implanted Bragg gratings in SOI waveguides" by M. P. Bulk et al.

"Tuning of the optical properties in photonic crystals made of macroporous silicon" by H.-S. Kitzerow et al.

"Silicon-based light sources for silicon integrated circuits" by L. Pavesi.

"Stress induced effects for advanced polarization control in silicon photonics components" by D.-X. Xu et al.

"Silicon photonic biosensors for "lab-on-a-chip" applications" by K. Zinoviev et al.

"Silicon nanocrystals: fundamental theory and implications for stimulated emission" by V. A. Belyakov et al.

"Quantum electrodynamic modeling of silicon-based active devices" by S. Shi et al.

"Three-dimensional silicon-Germanium nanostructures for CMOS compatible light emitters and optical interconnects" by L. Tsybeskov et al. 
"A new approach of electronics and photonics convergence on Si CMOS platform" by K. Wada.

"Slow light with photonic crystals for on-chip optical interconnects" by S. P. Anderson et al.

We thank the contributors for their very positive response to our request for papers and for providing informative overviews of their respective topics and new insights into forthcoming developments. We hope that this special issue will prove valuable to those working in silicon photonics and will help in advancing research in this field.

Pavel Cheben Richard Soref

David Lockwood Graham Reed 

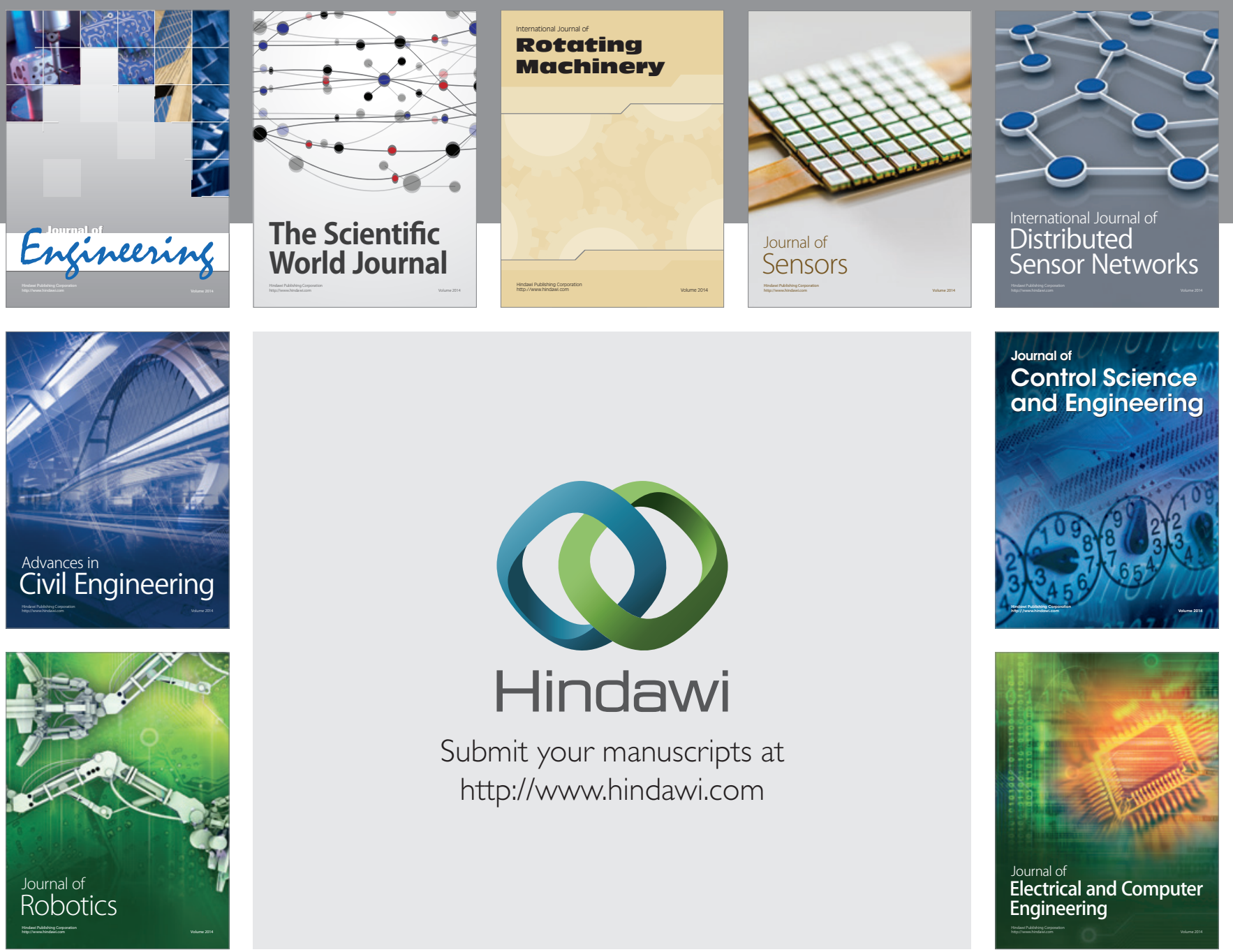

Submit your manuscripts at

http://www.hindawi.com
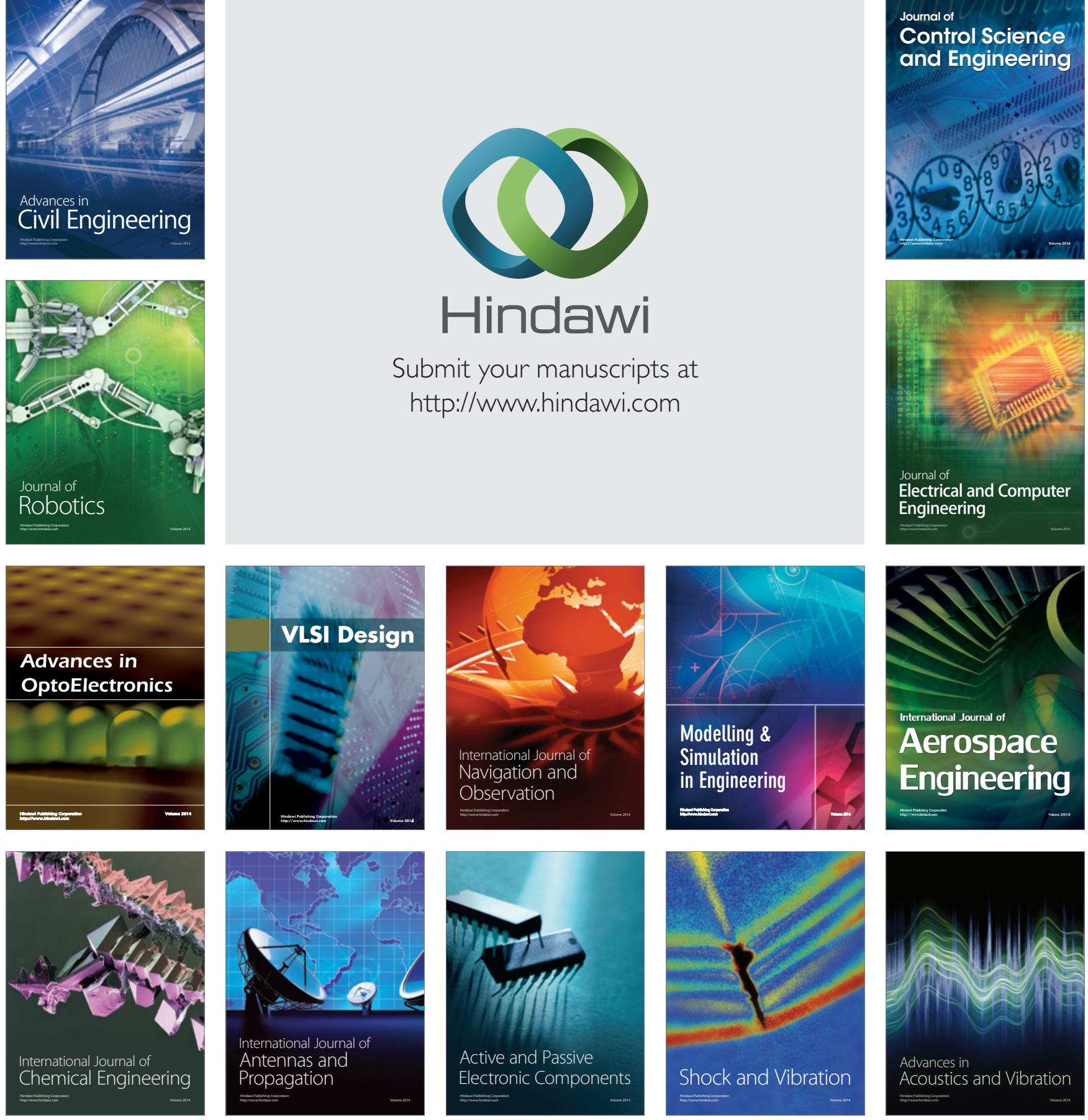